\title{
Ultrasonography on Detection of Massive Pulmonary Embolism as Cause of Cardiac Arrest
}

\section{Sandra Carvajal*, David De Paz and Darío Castaño}

Emergency Department, Fundación Valle del Lili, Cali, Colombia

*Corresponding author: Sandra Carvajal, Fundación Valle del Lili, Autopista Simón Bolívar, Carrera 98 No. 18-49, Cali, Colombia, Tel: (2) 6805757; Email: samikka2004@yahoo.com

Received date: June 12, 2017; Accepted date: June 26, 2017; Published date: July 03, 2017

Citation: Carvajal S, De Paz D, Castaño D. Ultrasonography on Detection of Massive Pulmonary Embolism as Cause of Cardiac Arrest. J Intensive \& Crit Care. 2017, 3:3

Copyright: (c) 2017 Carvajal S, et al. This is an open-access article distributed under the terms of the Creative Commons Attribution License, which permits unrestricted use, distribution, and reproduction in any medium, provided the original author and source are credited.

Keywords: Ultrasonography; Massive pulmonary embolism; Cardiac arrest

\section{Commentary}

Massive pulmonary embolism (MPE), defined as an acute Pulmonary Embolism (PE) with sustained hypotension, is estimated to occur in only $5 \%$ of cases of PE [1,2]. Of these $5 \%$ of cases, $10 \%$ to $20 \%$ of patients present with cardiac arrest, with a mortality rate of $65-90 \%[3,4]$. The guidelines of the European Society of Cardiology and the American Heart Association, on cardiopulmonary resuscitation, indicate the benefits of employing cardiopulmonary thrombolytic therapy if the patient is assumed to have a high-risk patient presenting with shock or cardiac arrest $[1,5,6]$.

In cases where the cause of cardiac arrest is PE, the use of thrombolysis and surgical or mechanical thrombectomy are considered reasonable treatment options [6]. However, it is difficult to correctly evaluate and select patients with PE as the one cause of cardiac arrest in real time [7]. There is no consensus as to the criteria (risk factors, signs and symptoms) which constitute a high-risk PE candidate for thrombolysis [6]. Yet, it is suggested that ultrasonography can provide support for medical emergencies, as this has proven to be a critical tool with which to identify the reversible causes of circulatory collapse, allowing physicians to make decisions in real time [6].

An emergency ultrasonography focused on the heart (FOCUS) is very useful to diagnose a massive PE in high-risk patients, and can also be very useful to guide management decisions. Although its usefulness has not been well established in cardiac arrest situations, it can be considered if a qualified professional is present and its use does not interfere with standard cardiopulmonary resuscitation $[4,6]$. The echocardiographic parameters describe a right ventricular overload whose signs are: growth of the ventricle and right atrium, hypokinesia of the free wall of the right ventricle (McConell's sign), flattening and paradoxical movement of the interventricular septum, with the left ventricle presenting in the form of a ' $D$ ' [4]. These signs are associated with an increase in the risk of mortality (up to two times higher).
In our experience, the use of bed-side ultrasonography with shock or cardiac arrest patients, has led to more accurate diagnosis and rapid decision of treatment for probable reversible causes of pulseless electrical activity, such as PE. Currently, we are driving an ambi-directional cardiac arrest registry in the emergency department and hope to describe the utility of ultrasound and survival of patients with PE, before and after its implementation.

\section{References}

1. Jaff MR, McMurtry MS, Archer SL, Cushman M, Goldenberg N, et al. (2011) Management of massive and sub massive pulmonary embolism, iliofemoral deep vein thrombosis and chronic thromboembolic pulmonary hypertension: A scientific statement from the american heart association. Circulation 123: 1788-1830.

2. Fengler BT, Brady WJ (2009) Fibrinolytic therapy in pulmonary embolism: an evidence-based treatment algorithm. Am J Emerg Med 27: 84-95.

3. Kasper W, Konstantinides S, Geibel A, Olschewski M, Heinrich F, et al. (1997) Management strategies and determinants of outcome in acute major pulmonary embolism: Results of a multicenter registry. J Am Coll Cardiol 30: 1165-1171.

4. Chowdhury MA, Moza A, Siddiqui NS, Bonnell M, Cooper CJ (2015) Emergent echocardiography and extracorporeal membrane oxygenation: Life-saving in massive pulmonary embolism. Heart Lung 44: 344-346.

5. Torbicki A, Perrier A, Konstantinides S, Agnelli G, Galie N, et al. (2008) Guidelines on the diagnosis and management of acute pulmonary embolism: The task force for the diagnosis and management of acute pulmonary embolism of the European Society of Cardiology (ESC). Eur Heart J 29: 2276-2315.

6. Lavonas EJ, Drennan IR, Gabrielli A, Heffner AC, Hoyte CO, et al. (2015) Part 10: Special circumstances of resuscitation. Circulation 132: S501-518.

7. Logan JK, Pantle H, Huiras P, Bessman E, Bright L (2014) Evidencebased diagnosis and thrombolytic treatment of cardiac arrest or peri-arrest due to suspected pulmonary embolism. Am J Emerg Med 32: 789-796. 